\title{
Interaction between Cholesterol and Chitosan in Langmuir Monolayers
}

\author{
Felippe J. Pavinatto, David S. dos Santos Jr., Osvaldo N. Oliveira Jr. \\ Instituto de Física de São Carlos, USP
}

\begin{abstract}
Chitosan incorporated in the aqueous subphase is found to affect the Langmuir monolayers of cholesterol, causing the surface pressure and the surface potential isotherms to become more expanded. The mean molecular area per cholesterol molecule in the condensed monolayer increases from $53 \AA^{2}$ in the absence of chitosan to $61 \AA^{2}$ for a concentration of $0.100 \mathrm{mg} / \mathrm{mL}$ of chitosan in the subphase. If additional chitosan is incorporated in the subphase, no change is noted, which points to saturation in the effects from chitosan. The interaction between chitosan and cholesterol probably occurs via hydrogen bonding. The monolayer expansion is also manifested in the monolayer morphology, as indicated by Brewster angle microscopy measurements, where larger cholesterol domains are visualized when chitosan is present in the subphase.
\end{abstract}

Keywords: Cholesterol, chitosan, Langmuir monolayers.

\section{Introduction}

Cholesterol is present in practically all cells of mammals, being essential for the functioning of some glandules and interacting with many compounds such as the lipids from the membrane and other cell components. When in excess, cholesterol may cause a number of health problems, and this has prompted researchers to search for ways of reducing it in the human body. Recently, chitosans have been suggested to be efficient in obesity treatments ${ }^{[1]}$, probably because they can interact with cholesterol. Chitosan is a biocompatible polysaccharide derived from chitin extracted from the exoskeleton of crustaceans and arthropods ${ }^{[2]}$, which has been employed in a number of applications, mainly in cosmetics ${ }^{[3]}$. With its biodegradability, chitosan can also be used in drug delivery systems ${ }^{[4]}$. It is clear, therefore, that studies of molecular-level interactions between chitosan and cholesterol are relevant. One possible way to investigate these interactions is to employ Langmuir monolayers that serve as simplified model for the cell membrane ${ }^{[5]}$. In most cases, phospholipid monolayers are used $^{[6-8]}$, but a number of studies exist with cholesterol, which also forms stable monolayers ${ }^{[9,10]}$ and interact with phospholipids ${ }^{[6,11-13]}$. With this approach, information can be obtained on a number of issues, including the drug location in the membrane and the cooperative response of the membrane to drugs ${ }^{[8]}$ and peptides ${ }^{[9]}$.

In this work we obtained Langmuir monolayers of cholesterol either on a pure water subphase or on a subphase containing dissolved chitosan. When chitosan is present, the system considered is a two-phase system with an aqueous subphase covered with an amphiphilic material (cholesterol with hydrophilic and hydrophobic groups) and interacting with a third component (chitosan, soluble in acidic medium). The monolayers were characterized with surface pressure, surface potential and Brewster angle microscopy (BAM) measurements. This study is a first step in the attempt to elucidate the activity of chitosan, which can also throw light into the interaction mechanisms between chitosan and cholesterol. It is remarked that Langmuir-Blodgett (LB) films of an amphiphilic chitosan were reported by $\mathrm{Li}$ et al. ${ }^{[14]}$, but to our knowledge there is no investigation of the effects from chitosan in the subphase on Langmuir monolayers.

\section{Experimental}

The cholesterol samples used in the experiments was 3ß-hydroxy-5-cholestene (Aldrich), employed as received. Chitosan was obtained from desacetylation of chitin extracted from shells of shrimps ${ }^{[15]}$ and the degree of acetylation was determined as $15 \%$ by nuclear magnetic resonance, according to the method of reference ${ }^{[16]}$. The chemical structures of these materials are shown in Figure 1.

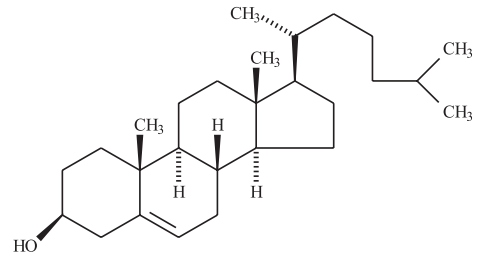

Cholesterol

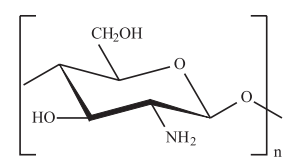

Chitosan
Figure 1. Cholesterol and chitosan structures

The Langmuir films were fabricated with a KSV 5000 Langmuir trough, equipped with a surface pressure sensor (Wilhelmy method) and a Kelvin probe to measure surface potential, housed in a class 10,000 clean room. Aliquots of a

Autor para correspondência: Osvaldo N. Oliveira Jr., Instituto de Física de São Carlos, USP, Av. Trabalhador São-carlense 400, CEP: 13560-970, São Carlos, SP. E-mail: chu@if.sc.usp.br 
chloroform (Mallinckrodt) cholesterol solution, $0.450 \mathrm{mg} / \mathrm{mL}$, were spread on an aqueous subphase with an acetate buffer, $\mathrm{pH}$ 3.5. The chitosan samples were dissolved in the same buffer solutions using concentrations between $0.050 \mathrm{mg} / \mathrm{mL}$ and $0.300 \mathrm{mg} / \mathrm{mL}$. Brewster angle microscopy (BAM) images were obtained with a BAM2 system from Nanofilm Technology (Germany), according to the experimental procedures described in reference ${ }^{[17]}$.

\section{Results and Discussion}

Langmuir films from pure cholesterol display surface pressure isotherms as shown in Figure 2A.

They are stable films, with a well-defined phase transition from the gaseous to the liquid condensed phase at $53 \AA^{2}$ per molecule. The collapse pressure is approximately $35 \mathrm{mN} / \mathrm{m}$, when an area of ca. $49 \AA^{2} / \mathrm{mol}$ is reached. When added to the subphase, chitosan has strong interaction with the cholesterol monolayer, and the isotherms become more expanded (Figures 2B to 2F) with the extrapolated area to zero pressure increasing. While the extrapolated area was $54 \AA^{2}$ per molecule for pure cholesterol, it increased to $59 \AA^{2}$ and $65 \AA^{2}$ for $0.050 \mathrm{mg} / \mathrm{mL}$ and $0.100 \mathrm{mg} / \mathrm{mL}$ chitosan concentrations in the subphase respectively, i.e. an increase between $9 \%$ and $20 \%$ in area for these concentrations. The expansion saturates when a concentration of $0.100 \mathrm{mg} / \mathrm{mL}$ of chitosan is employed. Further addition of chitosan in the subphase has no significant effect on the monolayer properties, with the maximum area per molecule being approximately $65 \AA^{2}$, as shown in isotherms $2 \mathrm{E}$ and $2 \mathrm{~F}$. Figure 3 shows the increase in mean molecular area with the chitosan concentration, where a clear saturation is observed for $0.100 \mathrm{mg} / \mathrm{mL}$ of chitosan in the subphase.

In addition, the surface pressure isotherms become less steep if chitosan is contained in the subphase. Chitosan affects the cholesterol monolayers, either by incorporating into the

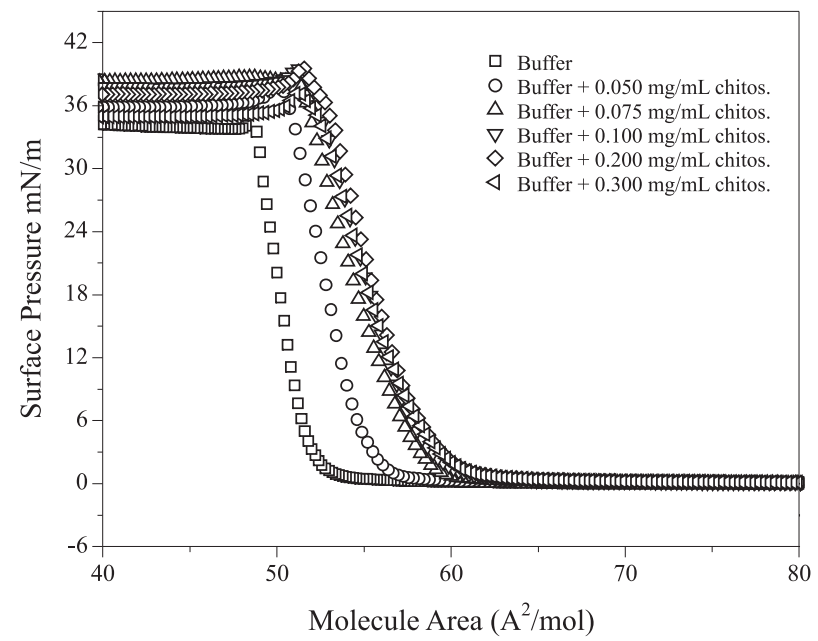

Figure 2. Surface pressure isotherms for cholesterol monolayers spread on the following subphases: $(\mathrm{OA})$ buffer $\mathrm{pH} 3.5 ;(\square \mathrm{B}),(\triangle \mathrm{C}),(\nabla \mathrm{D}),(\diamond \mathrm{E})$ and $(\triangleleft \mathrm{F})$ buffer $\mathrm{pH} 3.5+0.050 \mathrm{mg} / \mathrm{mL}, 0.075 \mathrm{mg} / \mathrm{mL}, 0.1 \mathrm{mg} / \mathrm{mL}, 0.2$ $\mathrm{mg} / \mathrm{mL}$ and $0.3 \mathrm{mg} / \mathrm{mL}$ of chitosan respectively.

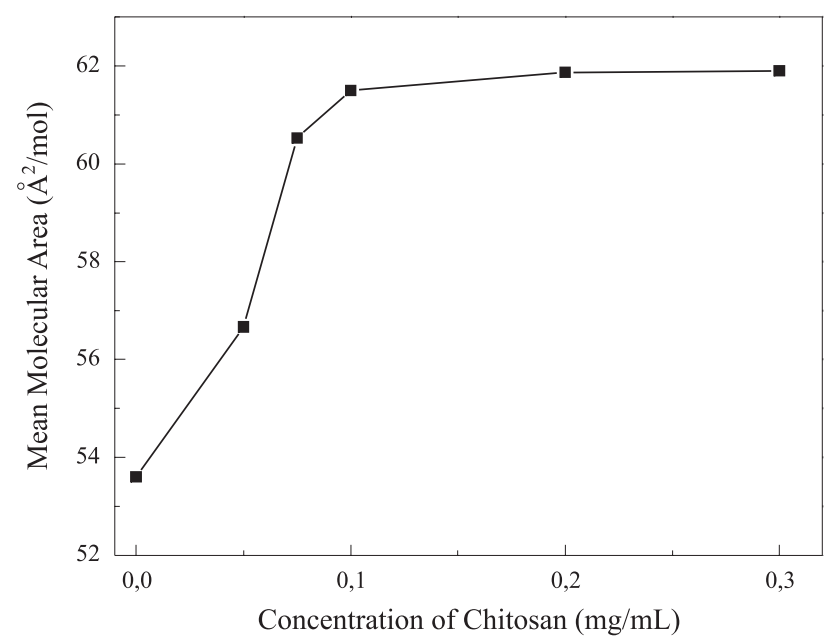

Figure 3. Mean area per molecule (extrapolated to zero pressure) vs. concentration of chitosan in the subphase

monolayer itself or by affecting the cholesterol conformation. In subsidiary experiments (results not shown) we observed that the cholesterol monolayers with chitosan in the subphase are stable for all chitosan concentrations employed in this study. For example, there is little change in area per molecule (less than 1\%) during several hours when the monolayer is kept at a fixed pressure of $18 \mathrm{mN} / \mathrm{m}$.

With regard to the surface potential isotherms, as expected they are more expanded for cholesterol monolayers spread on chitosan-containing subphases (Figure 4).

The maximum surface potential also increases slightly with the chitosan concentration. Note that the surface potential is already non-zero at the largest area per molecule plotted. In order to verify if a zero could be achieved - which means absence of large domains before compression starts - further experiments will be carried out with the compression starting at larger areas per cholesterol molecule. For aliphatic ${ }^{[18]}$ and aromatic compounds ${ }^{[19]}$ with well-defined headgroups, the measured surface potential can be related to the molecular dipole moments

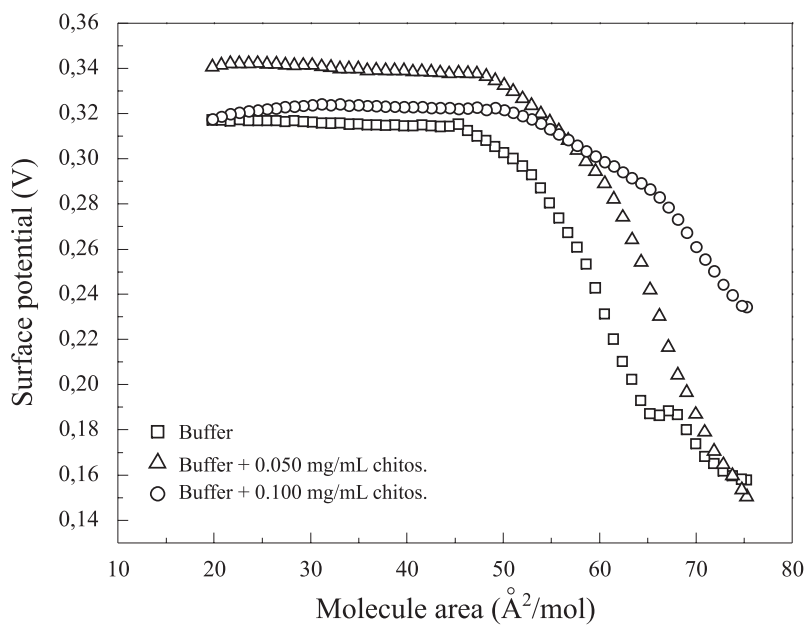

Figure 4. Surface potential isotherms for cholesterol monolayers spread on the following subphases: $(\square \mathrm{A})$ buffer $\mathrm{pH} 3.5 ;(\triangle \mathrm{B})$ buffer $\mathrm{pH} 3.5+$ $0.050 \mathrm{mg} / \mathrm{mL}$ of chitosan and (OC) buffer $\mathrm{pH} 3.5+0.100 \mathrm{mg} / \mathrm{mL}$ of chitosan. 


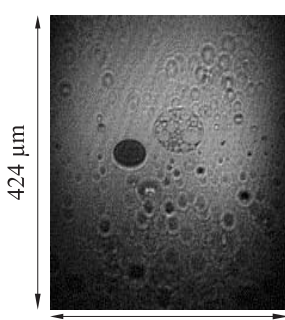

$281 \mu \mathrm{m}$

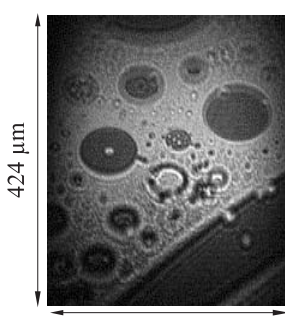

$281 \mu \mathrm{m}$

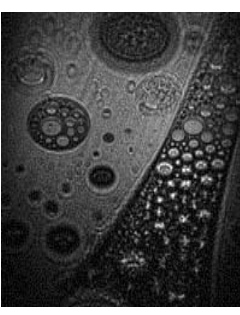

A)

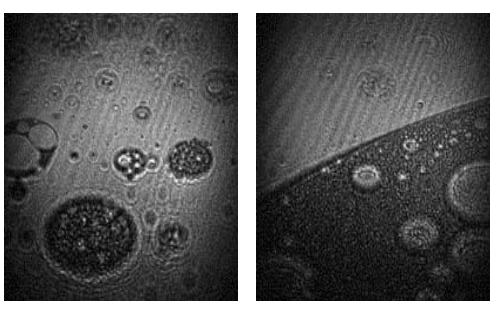

B)

)

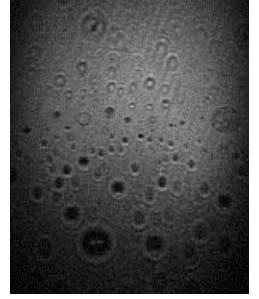

Figure 5. BAM images for cholesterol monolayers on the gaseous phase spread on (A) pH 3.5 buffer and (B) $\mathrm{pH} 3.5$ buffer $+0.050 \mathrm{mg} / \mathrm{mL}$ of chitosan

using the Demchak-Fort model ${ }^{[20]}$, which depicts the Langmuir monolayer as a 3-layer capacitor with dipole moments contributing at the air/monolayer and monolayer/water interfaces, in addition to reorientation of water molecules in the subphase due to the presence of the monolayer. For the cholesterol monolayers studied here, however, this quantitative treatment cannot be performed, as the precise orientation of the molecules at the interface is not known. It can be inferred, nevertheless, that chitosan does not alter the contribution to surface potential from the cholesterol dipole moments significantly, as demonstrated by the small changes in surface potential in Figure 4 upon addition of chitosan in the subphase.

The interaction between chitosan and cholesterol is also manifested in the morphology of the monolayers, as displayed in the images acquired with Brewster angle microscopy (BAM). Figure 5 shows images obtained for cholesterol monolayers spread on the $\mathrm{pH} 3.5$ buffer and another spread on a subphase containing chitosan.

Even though a number of features seen in the images may be related to experimental artifacts with interference fringes in several places, owing to the fluidity of the monolayer, it is clear that with chitosan in the subphase of the cholesterol domains become much larger, pointing to a considerable interaction between chitosan and cholesterol. It may be speculated that such interactions may occur via hydrogen bonding between hydroxyl groups present both in cholesterol and chitosan. The interaction between hydroxyl groups from cholesterol and the amine groups from chitosan is less important because at $\mathrm{pH}$ 3.5 almost all the amine groups are protonated and not available to establish hydrogen bonds. A quantification of the effects is not possible with the experiments carried out here because even though the degree of desacetylation of chitosan was 75 and the exact concentration of cholesterol are known, we cannot estimate how much of the chitosan comes to the monolayer interface to participate in the interaction. Experiments to establish the kinetics of the adsorption of chitosan are being carried out to determine such an amount.

\section{Conclusions}

The interaction between chitosan and cholesterol has been probed by spreading cholesterol monolayers on subphases containing chitosan. The latter was found to expand the cholesterol monolayer considerably and also affected the monolayer morphology as seen in BAM micrographs. There is a concentration of approximately $0.100 \mathrm{mg} / \mathrm{mL}$ at which the effect from chitosan is saturated. The interaction probably involves H-bonding between the hydroxyl groups of cholesterol and hydroxyl and amine groups from chitosan. One should also mention that with the results presented here we cannot establish whether the interaction between chitosan and cholesterol is specific or not.

\section{Acknowledgments}

This work was supported by CNPQ, CAPES, FAPESP, IMMP/MCT and PRP-USP.

\section{References}

1. Gallaher, D. D. - Agro Food Industry Hi-Tech, 14, p. 32-35 (2003).

2. "Chitin and chitosan - 4th International Conference on Chitin and Chitosan”, Elsevier Applied Science, Trondheim (1989).

3. Lang, G.; Wendel, H. \& Konrad, E. - US Patent 4,528,283

4. Thakkar, H.; Sharma, R.K.; Mishra, A.K.; Chuttani, K. \& Murthy, R.S.R. - J. of Pharmacy and Pharmacology, 56, p. 1091-1099 (2004).

5. Yokoi, H.; Hayashi, S. \& Kinoshita, T. - Progress in Polymer Science, 28, p. 341-357 (2003).

6. Sanchez, M.I.M. - “Interação de Moléculas Biologicamente Ativas com Filmes de Langmuir de Fosfolipídios" Tese de doutorado, Instituto de Física de São Carlos, USP, Brasil (2000).

7. Zheng, S.; Strzalka, J.; Jones, D.H.; Opella, S.J. \& Blasie, J.K. - Biophys. J., 84, p. 2393-2415 (2003).

8. Caetano, W.; Ferreira, M.; Tabak, M.; Sanchez, M.I.M.; Oliveira Jr., O.N.; Kruger, P.; Schalke, M. \& Losche, M. - Biophys. Chem., 91, p. 21-35 (2001).

9. Gomez-Serranillos, I.R.; Minones, J.; Dynarowicz-Latka, P. \& Iribarnegaray, E. - Langmuir, 20, p. 928-933 (2004).

10. Sparr, E.; Eriksson, L.; Bouwstra, J. A. \& Ekelund, K. Langmuir, 17, p. 164-172 (2001).

11. Kim, K.; Kim, C. \& Byun, Y. - Langmuir, 17, p. 50665070, (2001). 
12. Serfis, A.B.; Brancato, S. \& Fliesler, S.J. - Biochimica et Biophysica Acta, 1511, p. 341-348 (2001).

13. Brzozowska, I. \& Figaszewski, Z.A. - Biophysical Chemistry, 95, p. 173-179 (2002).

14. Li, M.; Xin, M. \& Miyashita T. - Polymer International, 51, p. 889-891 (2002)

15. Bough, W.; Salter, W.L. \& Perkinns, B.E. - Biotechnol. Bioeng., 20, p. 1931 (1978)

16. Signini, R. \& Campana Filho, S.P. - Polymer Bulletin, 42, p. $159-166(1999)$
17. Dos Santos Jr., D.S.; Pavinatto, F.J.; Balogh, D.T.; Misoguti, L.; Oliveira Jr., O.N. \& Mendonça, C.R. - J. Coll. and Interf. Sci., 276, p. 138 -142 (2004).

18. Oliveira Jr, O.N.; Taylor, D.M.; Lewis, T.J.; Salvagno, S. \& Stirling, C.J.M. - J. Chem. Soc.: Faraday Trans I, 85, p. 1009 (1989).

19. Dynarowicz-Latka, P.; Cavalli, A.; Silva Filho, D.A.; Milart, P.; Santos, M.C. \& Oliveira Jr., O.N. - Chem. Phys. Lett., 337, p. 11, (2001).

20. Demchack, R.J. \& Fort, T.J. - J. Coll. Interf. Sci., 46, p. 191 (1974). 